\title{
Association of Left Atrial Enlargement with Cortical Infarction in Subjects with Patent Foramen Ovale
}

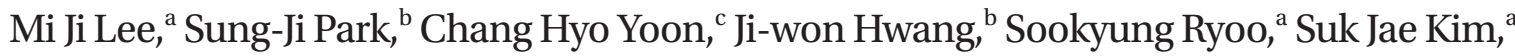 \\ Gyeong-Moon Kim, ${ }^{a}$ Chin-Sang Chung, ${ }^{a}$ Kwang Ho Lee, ${ }^{a}$ Oh Young Bang ${ }^{\text {a }}$ \\ aDepartment of Neurology, Samsung Medical Center, Sungkyunkwan University School of Medicine, Seoul, Korea \\ ${ }^{b}$ Department of Cardiology, Samsung Medical Center, Sungkyunkwan University School of Medicine, Seoul, Korea \\ 'Department of Radiology, Pusan National University Yangsan Hospital, Pusan National University School of Medicine, Yangsan, Korea
}

Background and Purpose Left atrial dysfunction has been reported in patients with patent foramen ovale (PFO). Here we investigated the role of left atrial dysfunction in the development of embolic stroke in patients with PFO.

Methods We identified consecutive patients with embolic stroke of undetermined sources except for PFO (PFO+ESUS). Healthy subjects with PFO served as controls (PFO+control). A stratified analysis by 10-year age group and an age- and sex- matching analysis were performed to compare echocardiographic markers between groups. In the PFO+ESUS group, infarct patterns of PFO-related stroke were determined (cortical vs. cortico-subcortical) and analyzed in correlation with left atrial function parameters

Results A total of 118 patients and 231 controls were included. The left atrial volume indices (LAVIs) of the PFO+ESUS patients were higher than those of the PF0+controls in age groups of 40-49, 5059, and 60-69 years $(P<0.001, P=0.003$, and $P=0.027$, respectively), and in the age- and sexmatched analysis $(P=0.001)$. In the PFO+ESUS patients, a higher $\left(>28 \mathrm{~mL} / \mathrm{m}^{2}\right) \mathrm{LAVI}$ was more associated with the cortical infarct pattern $(P=0.043$ for an acute infarction and $P=0.024$ for a chronic infarction, both adjusted for age and shunt amount). The degree of right-to-left shunting was not associated with infarct patterns, but with the posterior location of acute infarcts $(P=0.028)$.

Conclusions Left atrial enlargement was associated with embolic stroke in subjects with PFO. Left atrial physiology might contribute to the development of PFO-related stroke and need to be taken into consideration for optimal prevention of PFO-related stroke.

Keywords Echocardiography; Embolism; Left atrium; Paradoxical embolism; Patent foramen ovale; Stroke

\author{
Correspondence: Oh Young Bang \\ Department of Neurology, Samsung \\ Medical Center, Sungkyunkwan \\ University, 81 Irwon-ro, Gangnam-gu, \\ Seoul 06351, Korea \\ Tel: +82-2-3410-3599 \\ Fax: +82-2-3410-0052 \\ E-mail: ohyoung.bang@samsung.com \\ Received: April 12, 2016 \\ Revised: August 2, 2016 \\ Accepted: August 3, 2016
}

This study received grant support from the Korean Health Technology REtD Project, Ministry of Health \& Welfare, Republic of Korea (HI14C15310000 and HI13C1521).

The authors have no financial conflicts of interest.

\section{Introduction}

Patent foramen ovale (PFO) is prevalent in both the general population and stroke patients. Hence, it has been debated that PFO is a culprit of embolic stroke of undetermined source (ESUS). ${ }^{1}$ It was estimated that approximately one-third of PFOs discovered in cryptogenic stroke patients are likely to be incidental, and hence do not benefit from closure. ${ }^{2}$ The probability of having a PFO as a possible cause of stroke in cryptogenic stroke patients may vary according to patient characteristics. Younger patients without vascular risk factors are much more likely to have a PFO than patients with conventional risk factors. ${ }^{3}$ Several clinical and brain imaging features, including the 10-point Risk of Paradoxical Embolism (RoPE) score, have been suggested to determine a high-risk PFO. ${ }^{4}$

Paradoxical embolism has been considered as the mechanism 
of PFO-related stroke. However, there are many conflicting reports regarding the role of paradoxical embolism in PFO-related stroke. ${ }^{5-7}$ No association has been documented between shunt amount and stroke recurrence. ${ }^{8}$ Moreover, PFO closure did not prevent stroke. ${ }^{9}$ Clinical conditions such as deep vein thrombosis, a prolonged immobility/postoperative period, and the Valsalva maneuver were also not associated with embolism recurrence. ${ }^{10}$ Taken together, a mechanism other than paradoxical embolism may contribute to the development of embolic stroke in patients with PFO."

Recently, left atrial (LA) dysfunction has been reported in patients with PFO. ${ }^{12,13}$ Moreover, successful PFO closure reversed LA dysfunction. ${ }^{12}$ These findings suggest a role of LA dysfunction in the development of embolic stroke in patients with PFO. However, no study has investigated LA function in the risk stratification of PFO-related stroke.

We postulated that LA dysfunction could be involved in the development of an arterial embolism in patients with PFO. LA function comprises reservoir, conduit, and pump functions, all of which are dependent on left ventricular diastolic function. ${ }^{14,15}$ The LA volume index (LAVI), which is a biomarker of LA dysfunction that integrates the magnitude and duration of diastolic LV function, is strongly associated with cardiovascular disease and outcomes. ${ }^{14-17}$ Thus, we compared the LAVIs between ESUS patients with PFO and healthy subjects with PFO. To evaluate whether LA dysfunction is responsible for either PFO-related stroke or suggestive of occult atrial fibrillation (AF), we further analyzed the infarct patterns and correlated them with markers of $L A$ and LA appendage (LAA) dysfunction.

\section{Methods}

\section{Patient selection}

Using a prospective registry of consecutive patients who presented with acute ischemic stroke in the Samsung Medical Center from 2010 through 2014, we identified patients with PFO and ESUS (PFO+ESUS) using the following criteria: ${ }^{1}$ (1) an acute ischemic stroke was defined using diffusion-weighted imaging (DWI); (2) DWI showed an embolic stroke pattern (i.e., not restricted to the deep subcortical region); (3) a PFO was documented using appropriate diagnostic methods; and (4) no conventional stroke mechanisms (e.g., a relevant arterial stenosis of $>50 \%$, a high-risk cardioembolic source such as AF, small artery disease, etc.) were identified despite a comprehensive work-up. We excluded patients with transient ischemic attack and those with a single subcortical infarct that was restricted to a perforator territory.

\section{Controls}

Healthy subjects with PFO served as controls (PFO+controls). We prospectively recruited subjects who visited a health promotion center and participated in the institutional heart and brain program (the Comprehensive Brain and Heart Health [CBHH] program) from January 2011 to July 2014. The CBHH is a program operated by an integrated team of specialized neurologists, neuroradiologists, neurosurgeons, and cardiologists, providing comprehensive evaluation and management for primary and secondary stroke prevention. The CBHH standard protocol includes brain magnetic resonance imaging (MRI), MR angiogram, 12-lead electrocardiography, and 2-dimensional transthoracic echocardiography. For $\mathrm{CBHH}$ participants who have no ischemic lesions in their brain images, no conventional sources of stroke (major intra- or extracranial arterial stenosis $>50 \%$ ) or other evident cardioembolic sources, and no stroke or transient ischemic attack, as confirmed in an interview with the stroke neurologist (O.Y.B.), transcranial Doppler (TCD) shunt tests were performed for the presence of right-to-left shunting. Subjects with positive TCD shunt tests were included as "PFO+controls". A contrast echocardiogram was additionally conducted in 54 controls to measure septal movement. Among the PFO+controls, subjects were matched to individual PFO+ESUS cases based on gender and age. When more than 1 matched control was available, we selected 1 control at random. The Samsung Medical Center Institutional Review Board approved this study. All patients or patients' guardians provided written informed consent.

\section{Work-up}

Comprehensive evaluations for the detection of embolic sources were performed in the PFO+ESUS patients. All the included stroke patients underwent routine laboratory tests, 12lead electrocardiography, 2-D transthoracic echocardiography, 24-hour Holter monitoring, and/or > 72 hour telemonitoring, brain $\mathrm{MRI}$, and MR angiography. For patients who underwent Holter monitoring, the number of atrial premature complexes (APCs) was counted. Hemostatic markers of prothrombotic tendency were measured in patients younger than 50 years to exclude coagulopathies. Contrast transesophageal echocardiography (TEE) was the preferred method for detecting right-to-left shunting. In cases in which TEE was difficult to perform, a TCD shunt test was performed.

RoPE ${ }^{4}$ scores were determined in all patients. The location of ischemic lesions could not be assessed in PFO+controls, therefore we defined a modified RoPE score (excluding the cortical infarct) with a range of 0-9 to equally compare clinical risk scores between patients and controls. 


\section{Contrast echocardiography}

Two experts (S.J.P and J-w.H) who were blinded to the clinical information reviewed all of the contrast echocardiograms. The presence of PFO was defined as the appearance of microbubbles in the left atrium after intravenous injection of agitated saline, spontaneously and/or after a Valsalva or a cough maneuver. The atrial septal aneurysm was defined as a hypermobile septal excursion greater than $10 \mathrm{~mm}$.

\section{TCD shunt test protocol}

The protocol for the TCD shunt test has been described previously..$^{19}$ We determined right-to-left shunting based on the consensus criteria. ${ }^{20}$ The test was repeated twice at rest and twice during the Valsalva maneuver.

\section{Echocardiography parameters}

We collected echocardiographic parameters for all of the patients and control subjects. Biplane calculation of the LA volume was performed using LA areas, and lengths were measured from both the apical four- and two-chamber views, per previously established guidelines. ${ }^{21,22}$ Calculated LA volumes were indexed to the body surface area (LA volume index, LAVI). As most of our subjects were within the normal range, a normal LAVI cutoff with $28 \mathrm{~mL} / \mathrm{m}^{2}$ was used for the group comparisons. ${ }^{16,22}$ In the PFO+ESUS patients whose echocardiogram images were retrievable for re-analysis, the LA and LAA functions were measured with the total LA stroke volume, total LA ejection fraction, and LAA emptying velocity. Detailed methods of measurement are described in the supplementary material.

\section{Brain imaging analysis}

Investigators who were blinded to the clinical information (M.J.L. and O.Y.B.) measured the maximal diameters, multiplicities, and lesion distributions of acute infarcts. All patients had embolic (i.e., cortical or cortico-subcortical) infarcts. Infarct patterns were classified as cortical (less than a lobar involvement) and cortico-subcortical (lobar or territorial involvement) to respectively represent PFO-related and AF-related patterns. In the case of multiple infarctions, the lesion pattern was determined with the maximal lesion. Chronic ischemic lesions were defined as a high-signal-intensity lesion of $>3 \mathrm{~mm}$ in fluid attenuation inversion recovery sequence and a $\mathrm{T} 1$ hypointense signal in the corresponding area. These lesions were classified as cortical and cortico-subcortical infarcts using the same definition of acute infarctions. Periventricular and deep white matter hyperintensities were not considered as an infarction.

\section{Statistical analysis}

Chi-square tests, Fisher's exact tests, and Mann-Whitney tests were used to compare binary and continuous variables between groups. Possible confounders were adjusted using an analysis-ofcovariance (ANCOVA) test. To compare LAVI between groups, we performed a stratification analysis by each 10-year age group, and an age- and sex-matched analysis. For the analysis of matched samples, paired t-test and McNemar test were performed. Spearman's tests was performed to evaluate correlations between continuous variables. Statistical analyses were performed using SPSS version 18.0 (SPSS Inc., Chicago, IL, USA). A $P$-value $<0.05$ was considered to be statistically significant.

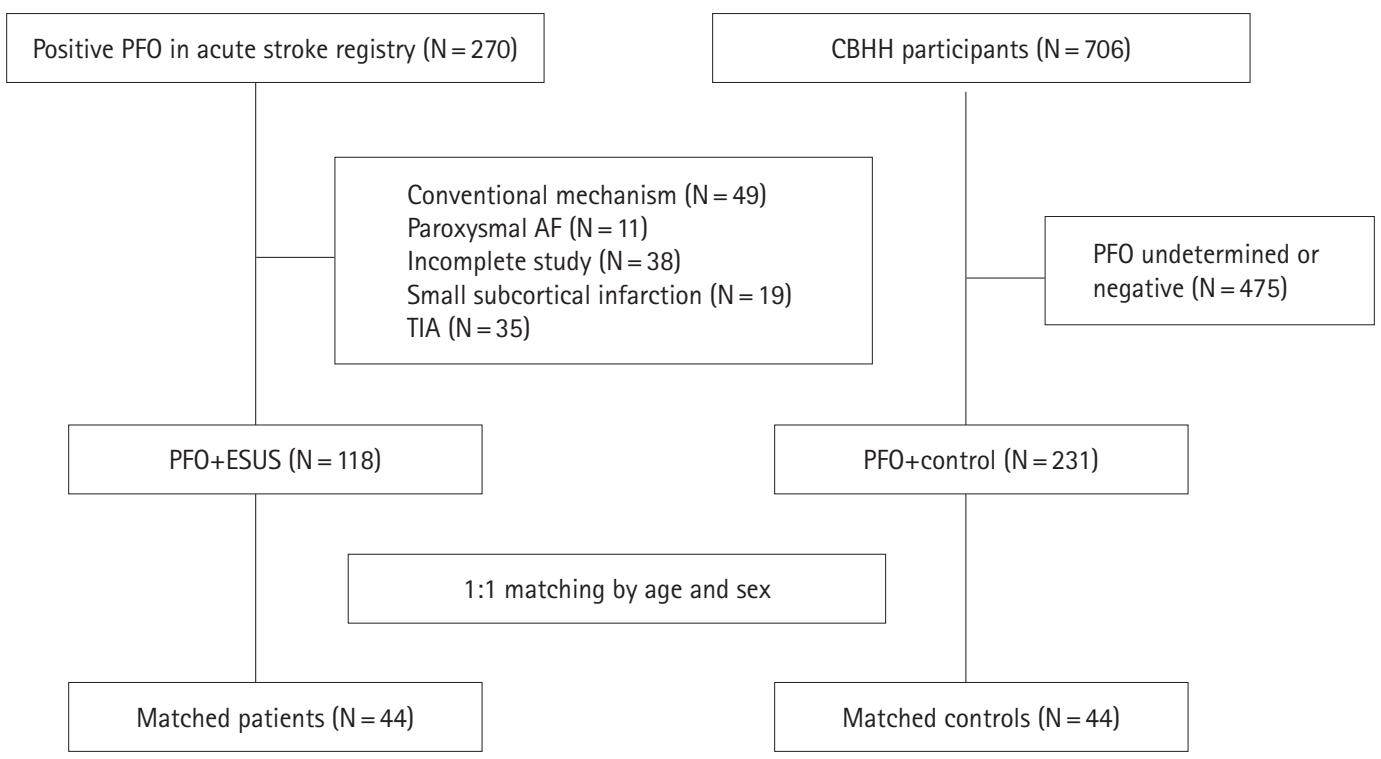

Figure 1. Study profile. PFO, patent foramen ovale; AF, atrial fibrillation; TIA, transient ischemic attack; ESUS, embolic stroke of unknown source; $\mathrm{CBHH}$, the Comprehensive Brain and Heart Health program. 


\section{Results}

The study profile is shown in Figure 1. A total of 118 patients were classified as PFO+ESUS, while 231 subjects without stroke served as PFO+controls. The clinical and PFO characteristics are presented in Table 1. The PFO+ESUS patients had significantly more males, current smokers, and younger patients than the PFO+control group (Table 1). The shunt amount was larger in the PFO+ESUS group than the PFO+control group $(P<0.001)$, albeit the diagnostic methods were different. The frequency of an atrial septal aneurysm was low in both groups ( $P=0.411$, Table 1$)$. Stratified comparisons of LAVI were made between all PFO+ESUS

Table 1. Characteristics of patients and controls

\begin{tabular}{|c|c|c|c|}
\hline & $\begin{array}{c}\text { PFO+ESUS } \\
(\mathrm{N}=118)\end{array}$ & $\begin{array}{c}\text { PFO+controls } \\
(\mathrm{N}=231)\end{array}$ & $P$ value \\
\hline Male gender & $83(70.3 \%)$ & $96(41.6 \%)$ & $<0.001$ \\
\hline Age (year) & $50(44.8-61.3)$ & 57 (51.0-64.0) & $<0.001$ \\
\hline $18-29$ & $4(3.4 \%)$ & $4(1.7 \%)$ & \\
\hline $30-39$ & $13(11.0 \%)$ & $5(2.2 \%)$ & \\
\hline $40-49$ & $41(34.7 \%)$ & $34(14.7 \%)$ & \\
\hline $50-59$ & $26(22.0 \%)$ & $91(39.4 \%)$ & \\
\hline $60-69$ & $21(17.8 \%)$ & $70(30.3 \%)$ & \\
\hline $70-79$ & $13(11.0 \%)$ & $26(11.3 \%)$ & \\
\hline $80-89$ & $0(0.0 \%)$ & $1(0.4 \%)$ & \\
\hline \multicolumn{4}{|l|}{ Risk factors } \\
\hline Hypertension & 47 (39.8\%) & $84(36.4 \%)$ & 0.527 \\
\hline Diabetes & $16(13.6 \%)$ & $21(9.1 \%)$ & 0.200 \\
\hline Hyperlipidemia & $29(24.6 \%)$ & $60(26.0 \%)$ & 0.777 \\
\hline Coronary artery disease & $9(7.6 \%)$ & $13(5.7 \%)$ & 0.487 \\
\hline Current smoking & $32(27.1 \%)$ & $28(12.1 \%)$ & $<0.001$ \\
\hline Prior stroke & $8(6.8 \%)$ & $0(0.0 \%)$ & $<0.001$ \\
\hline Atrial fibrillation & $0(0.0 \%)$ & $0(0.0 \%)$ & $>0.999$ \\
\hline RoPE score & $6(5-8)$ & NA & $N A$ \\
\hline Modified RoPE score* & $5(4-7)$ & $5(4-6)$ & 0.096 \\
\hline $\begin{array}{l}\text { Diagnostic methods for } \\
\text { shunting }\end{array}$ & & & $<0.001$ \\
\hline $\begin{array}{l}\text { Contrast transesophageal } \\
\text { echocardiogram }\end{array}$ & 95 (80.5\%) & & \\
\hline Transcranial Doppler & $23(19.5 \%)$ & $231(100.0 \%)$ & \\
\hline Shunt amount & & & $<0.001$ \\
\hline $1-10$ & $32(27.1 \%)$ & $103(44.5 \%)$ & \\
\hline $10-29$ & $35(29.7 \%)$ & $105(45.5 \%)$ & \\
\hline$>30$ & $51(43.2 \%)$ & $23(10.0 \%)$ & \\
\hline Atrial septal aneurysm ${ }^{+}$ & $2(1.7 \%)$ & $2(3.7 \%)$ & 0.411 \\
\hline
\end{tabular}

Values are presented as the number (percentage, \%) or median (interquartile range) unless otherwise specified.

PFO, patent foramen ovale; ESUS, embolic stroke of unknown source; NA, not assessed.

*The original RoPE (Risk of paradoxical embolism) score comprised of the history of hypertension, diabetes, prior stroke or transient ischemic attack, infarct lesion location (cortical lesion), and age group. Infarct lesion location was excluded in our modified RoPE score for balanced comparison between stroke and non-stroke subjects; ${ }^{\top}$ The presence of atrial septal aneurysm was assessed in all 118 PFO+ESUS patients and 54 PFO+controls who underwent the TTE shunt test. patients and all PFO+controls within each 10-year age group (Figure 2). LAVI was higher in the PFO+ESUS group than the PFO+controls within the age groups of 40-49, 50-59, and 6069 years in which enough numbers of patients and controls were

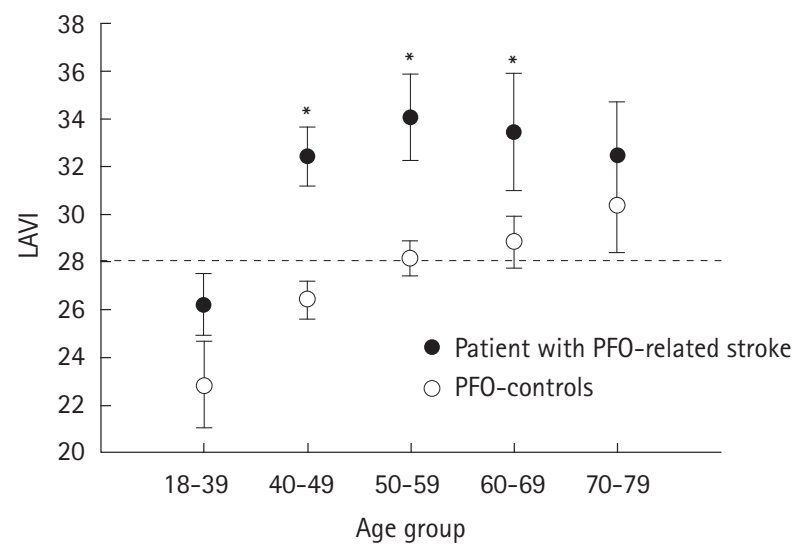

Figure 2. Left atrial volume indices (LAVIs) stratified by the 10-year age group. Ages of 18-29 and 30-39 were merged because of their small numbers. Error bars represent the standard error of the mean. PFO, patent foramen ovale. ${ }^{*}$ Indicates a statistical difference between groups.

Table 2. Clinical and echocardiographic findings of age- and sexmatched samples

\begin{tabular}{lccc}
\hline & $\begin{array}{c}\text { PFO+ESUS } \\
(\mathrm{N}=44)\end{array}$ & $\begin{array}{c}\text { PFO+Controls } \\
(\mathrm{N}=44)\end{array}$ & Pvalue* \\
\hline Age & $55.4(8.57)$ & $55.4(8.57)$ & $>0.999$ \\
Male sex & $29(65.9 \%)$ & $29(65.9 \%)$ & $>0.999$ \\
Risk factors & & & \\
$\quad$ Hypertension & $15(34.1 \%)$ & $16(36.4 \%)$ & 0.804 \\
$\quad$ Diabetes & $8(18.2 \%)$ & $6(13.6 \%)$ & 0.549 \\
Hyperlipidemia & $10(22.7 \%)$ & $11(25.0 \%)$ & $>0.999$ \\
Coronary artery disease & $3(6.8 \%)$ & $3(7.0 \%)$ & $>0.999$ \\
Current smoking & $11(25.0 \%)$ & $13(29.5 \%)$ & 0.754 \\
$\quad$ Prior stroke & $5(11.4 \%)$ & $0(0.0 \%)$ & 0.063 \\
Modified RoPE score ${ }^{\dagger}$ & $5.1(1.40)$ & $5.2(1.33)$ & 0.221 \\
LAVI (mL/m $\left.{ }^{2}\right)$ & $32.2(9.60)$ & $26.6(6.88)$ & 0.001 \\
$\quad>28\left(m L / m^{2}\right)$ & $28(63.6 \%)$ & $16(36.4 \%)$ & 0.004 \\
LAA emptying velocity $(\mathrm{cm} / \mathrm{s})$ & $61.0(13.84)$ & $\mathrm{NA}$ & $\mathrm{NA}$ \\
LA stroke volume $(\mathrm{mL})$ & $25.8(10.18)$ & $\mathrm{NA}$ & $\mathrm{NA}$ \\
LA ejection fraction $(\%)$ & $51.8(10.36)$ & $\mathrm{NA}$ & $\mathrm{NA}$ \\
LV end-diastolic dimension $(\mathrm{mm})$ & $48.8(4.32)$ & $48.7(4.22)$ & 0.900 \\
LV end-systolic dimension $(\mathrm{mm})$ & $29.4(3.92)$ & $29.5(4.52)$ & 0.940 \\
LA diameter (mm) & $35.3(5.32)$ & $36.7(4.70)$ & 0.234 \\
E/e' & $8.17(2.632)$ & $7.82(2.413)$ & 0.548 \\
LV ejection fraction & $63.4(5.17)$ & $63.8(5.57)$ & 0.724 \\
\hline
\end{tabular}

Values are presented as the mean (SD) or number (\%).

PFO, patent foramen ovale; ESUS, embolic stroke of undetermined source; RoPE, Risk of paradoxical embolism; LAVI, left atrial volume index; LAA, left atrial appendage; $L A$, left atrium; $L V$, left ventricle; NA, not assessed.

${ }^{*} P$ values were calculated from paired t-test or McNemar test; ${ }^{\text {TT }}$ The original RoPE (Risk of paradoxical embolism) score comprised of the history of hypertension, diabetes, prior stroke or transient ischemic attack, infarct lesion location (cortical lesion), and age group. Infarct lesion location was excluded in our modified RoPE score for balanced comparison between stroke and non-stroke subjects. 
included ( $P<0.001, P=0.003$, and $P=0.027$, respectively).

A total of 44 PFO+ESUS patients and 44 PFO+controls were included in the age- and sex-matched analysis. The demographics were comparable for both groups, with the exception of a higher prevalence of a previous stroke in the patient group $(P=$ 0.063 , Table 2). The PFO+ESUS patients showed higher LAVIs than controls (mean of $32.2(\mathrm{SD}=9.60) \mathrm{mL} / \mathrm{m}^{2}$ vs. 26.6 (6.88) $\mathrm{mL} / \mathrm{m}^{2}, P=0.001$ based on a paired t-test; Table 2). A high ( $>28$ $\mathrm{mL} / \mathrm{m}^{2}$ ) LAVI was documented in $63.6 \%$ of the PFO+ESUS patients and $36.4 \%$ of the PFO+controls $(P=0.004)$. There was no association between shunt amount and LAVI (Spearman correlation $P=0.346$ ). In addition, no interaction between shunt amount and LAVI on the group assignment (ESUS vs. controls) was noted ( $P$ for interaction $=0.405$ ).

In the PFO+ESUS patients, the acute lesion patterns included cortical (52.5\%), and cortico-subcortical (47.5\%) patterns. Typical examples are shown in Figure 3. The cortical lesion pattern was more common in the PFO+ESUS patients with a higher $\left(>28 \mathrm{~mL} / \mathrm{m}^{2}\right)$ LAVI than those with a lower LAVI $(P=0.043$, adjusted for age and shunt amount; Table 3). No other factors, including age, higher RoPE score, atrial septal aneurysm, redun- dancy, and the degree of right-to-left shunting were associated with a lesion pattern (data not shown). A higher LAVI was also associated with chronic cortical infarctions $(P=0.024$, adjusted

Table 3. Radiologic characteristics according to left atrial volume indices (LAVI) in patients with patent foramen ovale and embolic stroke of undetermined source

\begin{tabular}{|c|c|c|c|c|}
\hline & $\begin{array}{c}\quad L A V \mid \leq 28 \\
(N=41)\end{array}$ & $\begin{array}{c}\text { LAVI > 28 } \\
(\mathrm{N}=77)\end{array}$ & $P$ value & $\begin{array}{c}\text { Adjusted } \\
\qquad P^{*}\end{array}$ \\
\hline \multicolumn{5}{|c|}{ Acute infarct characteristics } \\
\hline Lesion pattern & & & 0.032 & 0.043 \\
\hline Cortical infarct & $16(39.0 \%)$ & $46(59.7 \%)$ & & \\
\hline $\begin{array}{l}\text { Cortico-subcortical } \\
\text { infarct }\end{array}$ & 25 (61.0\%) & $31(40.3 \%)$ & & \\
\hline Posterior involvement & $20(48.8 \%)$ & $46(60.5 \%)$ & 0.222 & 0.417 \\
\hline $\begin{array}{l}\text { Multiple territorial } \\
\text { involvement }\end{array}$ & $3(7.3 \%)$ & $11(14.3 \%)$ & 0.265 & 0.306 \\
\hline Multiplicity & $8(19.5 \%)$ & $25(32.5 \%)$ & 0.135 & 0.139 \\
\hline Diameter $>10 \mathrm{~mm}$ & $29(70.7 \%)$ & $49(64.5 \%)$ & 0.493 & 0.568 \\
\hline \multicolumn{5}{|c|}{ Chronic lesion characteristics } \\
\hline Cortical infarct & $6(14.6 \%)$ & $25(33.3 \%)$ & 0.030 & 0.024 \\
\hline $\begin{array}{l}\text { Cortico-subcortical } \\
\text { infarct }\end{array}$ & $0(0.0 \%)$ & $0(0.0 \%)$ & $>0.999$ & $>0.999$ \\
\hline
\end{tabular}

*Adjusted for age and shunt amount by analysis-of-covariance (ANCOVA) test.
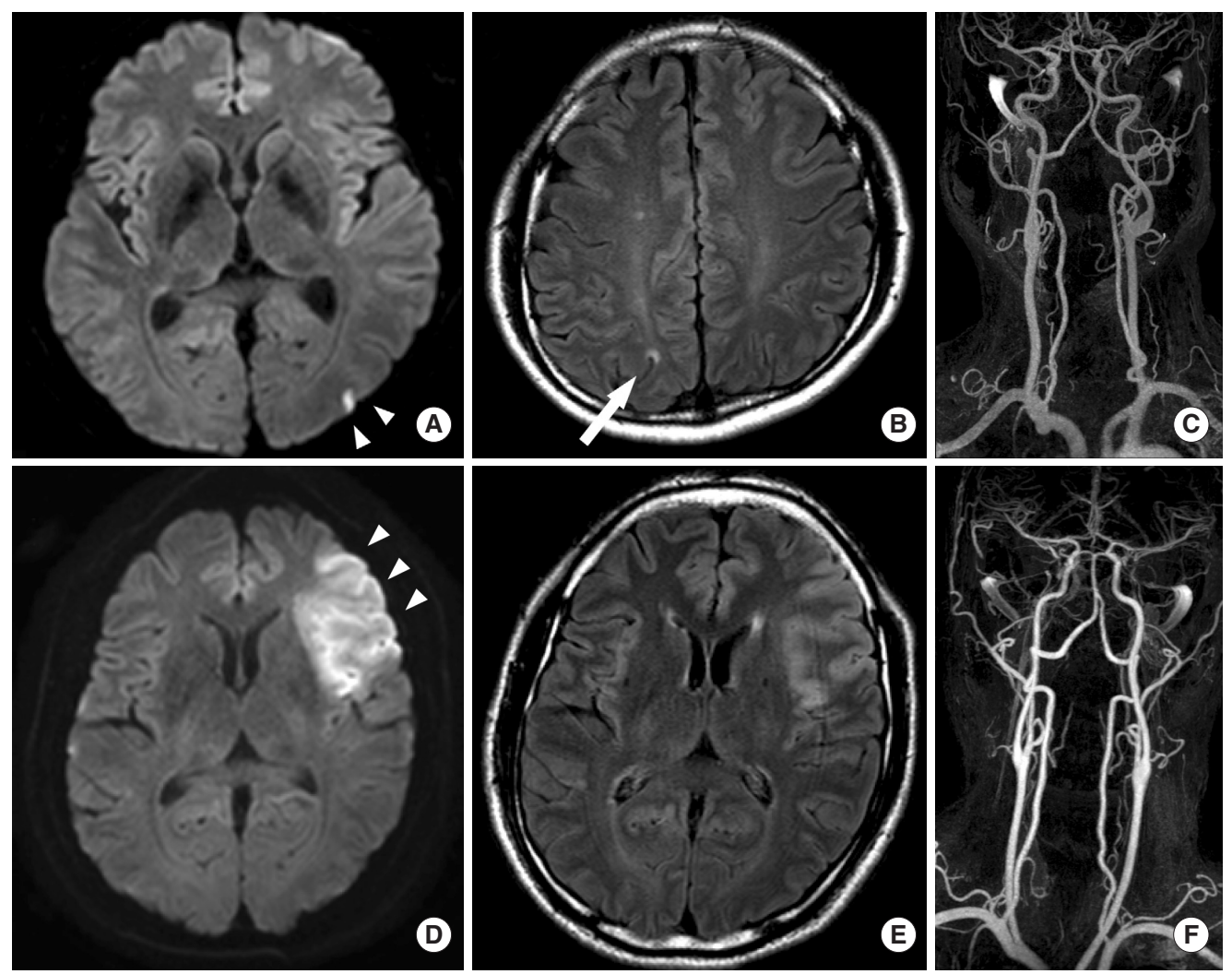

Figure 3. Typical examples of acute and chronic infarct patterns. (A-C) A 50-year-old man with a left atrial volume index of $44 \mathrm{~mL} / \mathrm{m}^{2}$. (A) A small cortical lesion is shown on a diffusion-weighted image. (B) A chronic cortical infarct is shown on fluid-attenuated inversion recovery images. (C) A normal MR angiography is shown. (D-F) A 46-year-old man with a left atrial volume index of $28.9 \mathrm{~mL} / \mathrm{m}^{2}$. (D) A large lobar lesion is shown on a diffusion-weighted image. (E) No chronic infarcts are shown on a fluid-attenuated inversion recovery image. (F) A normal MR angiography is shown. 
for age and shunt amount; Table 3). The RoPE score was not associated with the size, number, location, or pattern of the acute infarct. The degree of right-to-left shunting was only associated with the presence of posterior involvement ( $P$ for trend $=0.028$ ), but not with other patterns of acute or chronic infarcts.

In the PFO+ESUS patients, the LAA emptying velocity, LA stroke volume, and LA ejection fraction were within normal range in most cases (Table 2). The LAA emptying velocity and LA ejection fraction showed no correlation with the LAVI, while the LA stroke volume was positively correlated with the LAVI (Spearman's rho $=0.344, P=0.004)$. The LAA emptying velocities, $L A$ stroke volumes, and LA ejection fractions showed no correlation with the acute infarct lesion, in terms of maximal diameters, patterns, multiplicities, or sites of involvement (data not shown).

A total of 47 (39.8\%) PFO+ESUS patients underwent Holter monitoring, and 71 (60.2\%) received 72-hour in-hospital telemonitoring. Among the 47 Holter-monitored patients, no patients showed > 150 APCs, and 11 (23.4\%) patients showed no APCs, while 29 (61.7\%), $5(10.6 \%)$, and $2(4.3 \%)$ patients had $<25,25-100$, and 100-150 APCs, respectively.

\section{Discussion}

Our study showed that the PFO+ESUS patients had larger LA volumes than the PFO+controls. LA enlargement, but not a large amount of shunting, was associated with acute and chronic cortical ("PFO-related") infarctions. These findings suggest that the left atrium may play a role in the pathophysiology of PFO-related stroke.

Our data suggest that PFO-related stroke is associated with LA enlargement. LA enlargement was also associated with a higher frequency of chronic cortical infarcts, which could imply recurrent embolic stroke. An enlarged LA can precipitate thrombus formation. ${ }^{14}$ Whether the role of PFO on LA enlargement is causative, additive, or incidental has not been elucidated. One hypothesis is that a pathological PFO might contribute to atrial enlargement. Under normal conditions, PFO can allow only a rightto-left shunt because of the flap that covers it from the left side. However, when a distended LA stretches the foramen ovale or the flap valve is deficient, left-to-right shunting can occur. ${ }^{23}$ In this situation, left-to-right shunting can cause LA volume overload, and consequently, LA enlargement. In contrast, another possibility is that cardiac thrombus that is secondary to LA enlargement might be a determinant of stroke and PFO is additive or even incidental.

Rigatelli et al..$^{12}$ reported that a moderate to severe atrial septal aneurysm was associated with LA dysfunction. However, most of our subjects did not have an atrial septal aneurysm. Therefore, it is not likely that a higher LA volume is the result of an atrial septal aneurysm. Rather, increased LA volume is more likely to be a mediator in the development of embolic stroke in subjects with PFO. Taken together, our results indicate that a mild LA enlargement may precipitate incident and recurrent embolism from PFO in the absence of overt LA dysfunction.

In our study, a large shunt amount was found in the ESUS group. However, shunt amount was associated only with posterior circulation infarcts. Furthermore, shunt amount did not interact with LA enlargement in the development or pattern of stroke. Diverse pathophysiological mechanisms can be present in PFO-related stroke. Paradoxical embolism can be a mechanism in the development of a posterior infarction, and thrombus formation in the LA may be related to recurrent small cortical infarctions. Further large-scale studies that focus on stroke phenotype are needed to test our hypothesis.

As a major cause of embolic stroke, occult AF may exist in patients with ESUS and an incidental PFO. LA dysfunction could

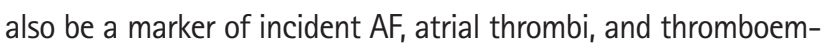
bolic risks of AF. ${ }^{1724}$ Rigatelli suggested that $L A$ functions of PFO patients were lower than normal and similar to AF patients. ${ }^{12}$ In addition, an increased incidence of interatrial block due to stretching of the interatrial septum was reported in patients with cryptogenic stroke and PFO, suggesting that atrial arrhythmia might underlie and mediate thrombus formation. ${ }^{13}$ In our study, however, neuroimaging, TEE, and Holter monitoring data showed patterns that were distinct from those of paroxysmal AF. ${ }^{25,26}$ The PFO+ESUS patients in our study had no markers of LAA dysfunction and infrequent APCs, reflecting a low possibility of occult $\mathrm{AF}^{25}$ We also analyzed an infarct pattern that enhances to infer stroke mechanisms. In the present study, a higher LAVI was associated with a small cortical pattern, suggesting a PFOrelated stroke ${ }^{27}$ without lobar or territorial infarctions, which are suggestive of a larger thromboembolism from a low-flow system such as $A F_{.}^{26,28}$ These findings suggest that $L A$ enlargement might be a factor in developing PFO-related stroke, rather than a marker of occult AF.

This study has several limitations. First, our controls were referred from the health promotion center and were willing to participate in the special program. The health-seeking nature of these controls might precipitate some unmeasured biases. In addition, the number of young (aged $<40$ years) controls was small, which led to insufficient case-control matching in young age groups. Second, for most of the subjects in the PFO+control group, a TCD shunt test was performed rather than TEE. The sensitivity and specificity of TCD tests for diagnosing a right to left shunt is compatible with TEE. ${ }^{29}$ However, interatrial septal movement was not assessed in most of our control subjects. Further- 
more, the opening size of the PFO was not measurable in most of our study subjects. Third, unrevealed conventional stroke mechanisms such as paroxysmal AF, and a mild degree of atherosclerotic stenosis that harbors unstable plaque may exist in our patients. ${ }^{26}$ Longer-term monitoring, such as implantable cardiac monitoring ${ }_{1}^{30,31}$ had not been available in Korea during our study period. Instead, we performed inpatient telemonitoring for $>72$ hours or Holter monitoring during admission, counted the number of APCs, and checked intermittent electrocardiography after discharge. ${ }^{25,32}$ As a result, we excluded 11 patients showing paroxysmal AF during follow-up. Lastly, the results of our study should be interpreted with caution because we only included relatively small samples from a single center. In addition, ethnic differences in the prevalence of right-to-left shunting may exist. ${ }^{33}$ Further studies involving different populations and larger cohorts are warranted.

\section{Conclusions}

In conclusion, LA enlargement might be a determinant of embolic stroke in subjects with PFO. LA enlargement was associated with a cortical infarct pattern and radiological evidence of stroke recurrence, even in the absence of an atrial septal aneurysm. These results would improve our understanding of the pathophysiology of PFO-related stroke.

\section{References}

1. Dennis M. Embolic stroke of undetermined source: a therapeutic target? Lancet Neurol 2014;13:344-346.

2. Alsheikh-Ali AA, Thaler DE, Kent DM. Patent foramen ovale in cryptogenic stroke: incidental or pathogenic? Stroke 2009;40: 2349-2355.

3. Kent DM, Thaler DE. Is patent foramen ovale a modifiable risk factor for stroke recurrence? Stroke 2010;41(suppl):S26-30.

4. Kent DM, Ruthazer R, Weimar C, Mas JL, Serena J, Homma S, et al. An index to identify stroke-related vs incidental patent foramen ovale in cryptogenic stroke. Neurology 2013;81:619625.

5. Meier B, Kalesan B, Mattle HP, Khattab AA, Hildick-Smith D, Dudek $D$, et al. Percutaneous closure of patent foramen ovale in cryptogenic embolism. N Engl J Med 2013;368:1083-1091.

6. Carroll JD, Saver JL, Thaler DE, Smalling RW, Berry S, MacDonald LA, et al. Closure of patent foramen ovale versus medical therapy after cryptogenic stroke. N Engl J Med 2013;368: 1092-1100.

7. Furlan AJ, Reisman M, Massaro J, Mauri L, Adams H, Albers $\mathrm{GW}$, et al. Closure or medical therapy for cryptogenic stroke with patent foramen ovale. N Engl J Med 2012;366:991-999.

8. Serena J, Marti-Fabregas J, Santamarina E, Rodriguez JJ, Perez-Ayuso MJ, Masjuan J, et al. Recurrent stroke and massive right-to-left shunt: results from the prospective Spanish multicenter (CODICIA) study. Stroke 2008;39:3131-3136.

9. Alushi B, Biasco L, Orzan F, Omede P, Sciuto F, Moretti C, et al. Patent foramen ovale treatment strategy: an Italian large prospective study. J Cardiovasc Med (Hagerstown) 2014;15:761768.

10. Kitsios GD, Lasker A, Singh J, Thaler DE. Recurrent stroke on imaging and presumed paradoxical embolism: a cross-sectional analysis. Neurology 2012;78:993-997.

11. Bang OY, Lee MJ, Ryoo S, Kim SJ, Kim JW. Patent Foramen Ovale and Stroke-Current Status. J Stroke 2015;17:229-237.

12. Rigatelli G, Aggio S, Cardaioli P, Braggion G, Giordan $M$, Dell'avvocata $F$, et al. Left atrial dysfunction in patients with patent foramen ovale and atrial septal aneurysm: an alternative concurrent mechanism for arterial embolism? JACC Cardiovasc Interv 2009;2:655-662.

13. Cotter PE, Martin PJ, Pugh PJ, Warburton EA, Cheriyan J, Belham M. Increased incidence of interatrial block in younger adults with cryptogenic stroke and patent foramen ovale. Cerebrovasc Dis Extra 2011;1:36-43.

14. Hoit BD. Left atrial size and function: role in prognosis. $J \mathrm{Am}$ Coll Cardiol 2014;63:493-505.

15. Russo C, Jin Z, Homma S, Rundek T, Elkind MS, Sacco RL, et al. Left atrial minimum volume and reservoir function as correlates of left ventricular diastolic function: impact of left ventricular systolic function. Heart 2012;98:813-820.

16. Alsaileek AA, Osranek M, Fatema K, McCully RB, Tsang TS, Seward JB. Predictive value of normal left atrial volume in stress echocardiography. J Am Coll Cardiol 2006;47:10241028.

17. Leung DY, Boyd A, Ng AA, Chi C, Thomas L. Echocardiographic evaluation of left atrial size and function: current understanding, pathophysiologic correlates, and prognostic implications. Am Heart J 2008;156:1056-1064.

18. Jeong WK, Choi JH, Son JP, Lee S, Lee MJ, Choe YH, et al. Volume and morphology of left atrial appendage as determinants of stroke subtype in patients with atrial fibrillation. Heart Rhythm 2016;13:820-827.

19. Kim SJ, Shin HY, Ha YS, Kim JW, Kang KW, Na DL, et al. Paradoxical embolism as a cause of silent brain infarctions in healthy subjects: the ICONS study (Identification of the Cause of Silent Cerebral Infarction in Healthy Subjects). Eur J Neurol 2013;20:353-360.

20. Jauss $M$, Zanette $E$. Detection of right-to-left shunt with ultrasound contrast agent and transcranial Doppler sonography. 
Cerebrovascular diseases 1999;10:490-496.

21. Lang RM, Badano LP, Mor-Avi V, Afilalo J, Armstrong A, Ernande $L$, et al. Recommendations for cardiac chamber quantification by echocardiography in adults: an update from the American Society of Echocardiography and the European Association of Cardiovascular Imaging. J Am Soc Echocardiogr 2015;28:1-39 e14.

22. Lang RM, Bierig M, Devereux RB, Flachskampf FA, Foster $E$, Pellikka PA, et al. Recommendations for chamber quantification: a report from the American Society of Echocardiography's Guidelines and Standards Committee and the Chamber Quantification Writing Group, developed in conjunction with the European Association of Echocardiography, a branch of the European Society of Cardiology. J Am Soc Echocardiogr 2005;18:1440-1463.

23. Hasan A, Parvez A, Ajmal M. Patent Foramen Ovale-Clinical Significance. J Indian Acad Clin Med 2004;5:339-344.

24. Agmon Y, Khandheria BK, Gentile F, Seward JB. Echocardiographic assessment of the left atrial appendage. J Am Coll Cardiol 1999;34:1867-1877.

25. Gladstone DJ, Dorian P, Spring M, Panzov V, Mamdani M, Healey JS, et al. Atrial Premature Beats Predict Atrial Fibrillation in Cryptogenic Stroke: Results From the EMBRACE Trial. Stroke 2015;46:936-941.

26. Bang OY, Ovbiagele B, Kim JS. Evaluation of cryptogenic stroke with advanced diagnostic techniques. Stroke 2014;45:11861194.
27. Kim BJ, Sohn H, Sun BJ, Song JK, Kang DW, Kim JS, et al. Imaging characteristics of ischemic strokes related to patent foramen ovale. Stroke 2013;44:3350-3356.

28. Fujimoto S, Toyoda K, Jinnouchi J, Yasaka M, Kitazono T, Okada $Y$. Differences in diffusion-weighted image and transesophageal echocardiographical findings in cardiogenic, paradoxical and aortogenic brain embolism. Cerebrovasc Dis 2011;32:148154.

29. Mojadidi MK, Roberts SC, Winoker JS, Romero J, GoodmanMeza D, Gevorgyan $R$, et al. Accuracy of transcranial Doppler for the diagnosis of intracardiac right-to-left shunt: a bivariate meta-analysis of prospective studies. JACC Cardiovasc Imaging 2014;7:236-250.

30. Gladstone DJ, Spring M, Dorian P, Panzov V, Thorpe KE, Hall J, et al. Atrial fibrillation in patients with cryptogenic stroke. N Engl J Med 2014;370:2467-2477.

31. Sanna T, Diener HC, Passman RS, Di Lazzaro V, Bernstein RA, Morillo $C A$, et al. Cryptogenic stroke and underlying atrial fibrillation. N Engl J Med 2014;370:2478-2486.

32. Douen AG, Pageau N, Medic S. Serial electrocardiographic assessments significantly improve detection of atrial fibrillation 2.6-fold in patients with acute stroke. Stroke 2008;39:480482.

33. Rodriguez CJ, Homma S, Sacco RL, Di Tullio MR, Sciacca RR, Mohr JP. Race-ethnic differences in patent foramen ovale, atrial septal aneurysm, and right atrial anatomy among ischemic stroke patients. Stroke 2003;34:2097-2102. 


\section{Supplementary material}

To obtain left atrial (LA) areas, 2-D volumetric measurements were performed based on tracings of the blood-tissue interface on apical four- and two-chamber views at the end-diastole and end-systole. End-diastolic areas were digitized from apical biplane echo images that were coincident with the beginning of the Q-wave on the electrocardiogram. End-systolic areas were digitized from video frames near the end of the T-wave with a maximum inward motion of the left ventricular walls. The LA volumes were calculated at the end-diastole and end-systole using Echopac software (GE Vingmed, Milwaukee, WI, USA) with the modified Simpson's rule.' The LA stroke volume was defined as the difference between the LA end-systolic and end-diastolic volumes, and the LA ejection fraction was defined as the proportion of the LA stroke volume to the LA end-systolic volume. ${ }^{2}$ The LA appendage emptying velocity was measured using a pulsed Doppler wave, which was placed 1 to $2 \mathrm{~cm}$ from the LA appendage orifice within the chamber. ${ }^{3}$ Both inter-rater and intra-rater reliability for measurements were excellent for the LA stroke volume (intraclass correlation coefficient [ICC] $=0.919$ and 0.974 , respectively, $P<0.001)$, LA ejection fraction (ICC $=0.907$ and 0.893, respectively, $P<0.001)$ and the $L A$ appendage emptying velocity ( $I C C=0.936$ and 0.960 , respectively, $P<0.001)$. Normal values for the $L A$ stroke volume, $L A$ ejection fraction, and LA ap- pendage emptying velocity were determined in accordance with current recommendations. ${ }^{1,3,4}$

\section{References}

1 Lang RM, Badano LP, Mor-Avi V, Afilalo J, Armstrong A, Ernande $L$ et al. Recommendations for cardiac chamber quantification by echocardiography in adults: an update from the American Society of Echocardiography and the European Association of Cardiovascular Imaging. J Am Soc Echocardiogr 2015;28:1-39.

2 Russo C, Jin Z, Homma S, Rundek T, Elkind MS, Sacco RL et al. Left atrial minimum volume and reservoir function as correlates of left ventricular diastolic function: impact of left ventricular systolic function. Heart 2012; 98:813-20.

3 Hahn RT, Abraham T, Adams MS, Bruce CJ, Glas KE, Lang RM et al. Guidelines for performing a comprehensive transesophageal echocardiographic examination: recommendations from the American Society of Echocardiography and the Society of Cardiovascular Anesthesiologists. J Am Soc Echocardiogr 2013; 26:921-64.

4 Aune E, Baekkevar M, Roislien J, Rodevand O, Otterstad JE. Normal reference ranges for left and right atrial volume indexes and ejection fractions obtained with real-time three-dimensional echocardiography. Eur J Echocardiogr 2009;10:738-44. 